\title{
Primary care-based audit on the review of oral nutritional supplement prescribing patterns on the Wirral
}

\author{
A. M. Wigley, M. Kent and R. J. S. Costa \\ Wirral PCT Nutrition and Dietetics Services, St Catherine's Hospital, Church Road, Birkenhead CH42 OLQ, UK
}

The incidences, causes and consequences of disease-related malnutrition (DRM) in the community have been well documented ${ }^{(1-3)}$. Appropriate dietetic intervention is required at an early stage of DRM to reduce negative outcomes, costs, duration of medical treatments and improve patient quality of life. Prescription of oral nutritional supplements (ONS) has been indicated as an initial part of dietary intervention for DRM and has been reported to improve nutritional status ${ }^{(4)}$. However, long-term efficacy of ONS prescription in primary care has not been definitively established. In recent years there has been an increase in the prescription and spending of ONS within the Wirral Community.

The aim of the present audit was to identify ONS prescription patterns in the Wirral Primary Care setting and assess appropriateness of prescription initiation and continuation.

Between the period of January 2003 and March 2007, 497 adult patients from forty-four general practitioner (GP) practices were audited, having been referred to Wirral Primary Care Dietetic Service for oral nutrition support and were receiving ONS. Patients were screened and appropriate consultation arranged. Full nutritional assessment was performed by a registered dietitian. Individualised nutritional care plans were formulated, including provision of food-first and food fortification dietary advice where appropriate. ONS were continued, discontinued or altered as appropriate and need for further review was also assessed.

Of the 497 patients referred for oral nutrition support, ONS prescription was predominantly initiated by acute-based dietitians and GP (Table). No monitoring or review of initiated ONS prescription was evident in $35 \%$ of the audited patients. For the $65 \%$ of patients who were monitored and reviewed, it was mainly primary care dietitians, and to a less extent acute-based dietitians and GP, who were responsible. No review of prescribed ONS was evident when ONS prescription request was initiated by acute-based medical staff, other health professionals and from unknown origin.

Table. Initiation of ONS for patients ( $n$ 497) referred to primary care nutrition and dietetic services ( $\%$ total)

\begin{tabular}{lcccc}
\hline $\begin{array}{l}\text { Primary care } \\
\text { dietitians }\end{array}$ & $\begin{array}{c}\text { Acute-based } \\
\text { dietitians }\end{array}$ & GP & $\begin{array}{c}\text { Acute-based } \\
\text { medical staff }\end{array}$ & $\begin{array}{c}\text { Other health } \\
\text { professionals }\end{array}$ \\
\hline 15 & 35 & 30 & 9 & Unknown \\
\hline
\end{tabular}

On review of the nutritional care plans of 306 patients by a primary care dietitian, it was considered that $36 \%$ of patients would benefit from continued prescription, whilst $50 \%$ of patients did not require ONS to meet nutritional requirements and $14 \%$ of patients had their ONS prescription reduced. The majority of discontinued ONS prescriptions were initiated by acute-based dietitians and GP. All ONS prescriptions initiated by acute-based medical staff, unknown and other health professionals were seen to be inappropriate and discontinued.

The current audit indicates that ONS prescriptions commenced in secondary care and by GP are more likely not to be appropriate, monitored and/or may require partial or total modification during primary care dietetic review. The observed outcomes provide a practical base for the need to develop ONS prescription and review guidelines in primary care; thus, improving the transfer of patients on ONS from secondary care to primary care.

1. Green CJ (1999) Clin Nutr 18, Suppl. 2, 3-28.

2. Stratton RJ \& Elia M (1999) Clin Nutr 18, Suppl. 2, 29-84.

3. Milne AC, Avenell A \& Potter J (2006) Ann Intern Med 144, 37-48.

4. Stratton RJ (2005) Proc Nutr Soc 64, 325-333. 